\title{
DETERMINAÇÃO DO TEOR DE ETANOL ANIDRO EM GASOLINA POR CALIBRAÇÃO MULTIVARIADA UTILIZANDO ESPECTROSCOPIA NO INFRAVERMELHO MÉDIO
}

\author{
Vitoria Hagemann Cauduro ${ }^{1}$, Franccesca Fornasier ${ }^{1}$, Aline Teichmann ${ }^{2}$, Adilson Ben da Costa, ${ }^{2,3 *}$ \\ ${ }_{1}$ Curso em Química Industrial - Universidade de Santa Cruz do Sul (UNISC) - Santa Cruz do Sul, RS - Brasil \\ 2 Programa de Pós-graduação em Sistemas e Processos Industriais - Universidade de Santa Cruz do Sul (UNISC) - Santa Cruz do \\ Sul, $R S$ - Brasil \\ 3 Programa de Pós-graduação em Tecnologia Ambiental - Universidade de Santa Cruz do Sul (UNISC) - Santa Cruz do Sul, RS - \\ Brasil
}

*E-mail: adilson@unisc.br

Recebido em: 02/01/2017 Aceito em: 02/06/2017

\section{RESUMO}

O objetivo principal deste estudo foi desenvolver um modelo multivariado de calibração por quadrados parciais (PLS) para quantificação do teor de etanol anidro em amostras comerciais de gasolina, utilizando um software de quimiometria gratuito. Para isso, espectros de infravermelho médio das amostras foram adquiridos em uma atividade experimental de estudantes de graduação em Química da UNISC, para melhorar a compreensão dos métodos de calibração multivariada. O modelo de calibração PLS apresentou resultados semelhantes aos obtidos pelo método de referência (NBR:13992) (p > 0,05), com valores de exatidão entre 97,8 e 108,2\%. Além disso, a metodologia desenvolvida neste estudo se destaca por consumir até 25 vezes menos amostra $(2 \mathrm{~mL})$, o que resulta em menor geração de resíduos e maior frequência de análise ( $>30$ amostras por hora), baixo consumo de energia $\left(340 \mathrm{Wh}^{-1}\right)$ e na dispensa do uso de reagentes.

Palavras-chave: Quimiometria. PLS. Etanol. Gasolina.

\section{Introdução}

A constante evolução da instrumentação analítica e da quimiometria tem permitido aos químicos a aquisição, exploração e análise de grandes volumes de informações, notadamente impossível num passado recente [1]. Nas últimas décadas, a utilização de métodos espectrométricos aliados aos métodos matemáticos tem contribuído significativamente para o desenvolvimento de novas metodologias qualitativas e quantitativas de análise, com menor consumo de reagentes, materiais, energia e menor geração de resíduos [2].

A quimiometria emprega métodos matemáticos e estatísticos para planejar ou selecionar experimentos de forma otimizada e para fornecer o máximo de informação química com a análise dos dados obtidos [2, 3]. Os estudos em quimiometria desenvolvidos no Brasil podem ser agrupados em três áreas principais: a) planejamento e otimização de experimentos; b) reconhecimento de padrões (métodos de análise exploratória e classificação) e c) calibração multivariada. Além disso, segundo Rinnan, et al. [4], o pré-processamento dos dados pode ser considerado uma área à parte da quimiometria.
Entre os diferentes métodos de calibração multivariada, destaca-se a regressão por mínimos quadrados parciais (PLS), que utiliza a técnica de análise de componentes principais (PCA) para reduzir o tamanho do conjunto de dados antes de correlacionar os dados espectrais e as propriedades de interesse $[5,6]$. Na construção de um modelo de calibração multivariada, uma matriz $\mathrm{X}_{(\mathrm{ixj})}$ é obtida a partir dos espectros de infravermelho das amostras de calibração e, em um vetor $\mathrm{Y}_{(\mathrm{ixj})}$, são adicionados os valores das variáveis de interesse, em que "i" corresponde ao número de amostras e "j" ao número de variáveis [7].

Diferente de outros modelos, no método de PLS a informação da propriedade de interesse é incluída no cálculo das variáveis latentes, sendo mais eficiente para capturar informações relevantes para a construção do modelo de calibração [2].

A espectroscopia no infravermelho, associada à calibração multivariada, tem se destacado como uma ferramenta analítica poderosa no desenvolvimento de metodologias analíticas para a avaliação qualitativa e quantitativa de diferentes tipos de materiais, tais como: derivados do petróleo [8]; extratos vegetais [9]; medicamentos [5, 10]; biocombustíveis [11] e alimentos [6, $12]$. 
Desta forma, este estudo descreve um experimento de desenvolvimento em sala de aula, aplicado na determinação dos teores de etanol anidro combustível em diferentes amostras de gasolina. Para isso, foi utilizada a espectroscopia no infravermelho médio aliada à calibração multivariada como um método alternativo ao método de referência descrito na norma NBR 13992:2015.

$\mathrm{O}$ etanol anidro é adicionado à composição da gasolina na proporção de $25 \%$ para gasolina Premium e $27 \%$ para gasolina comum, conforme a Portaria $\mathrm{n}^{\mathrm{o}} 75$ do Ministério da Agricultura, Pecuária e Abastecimento [13], agindo como um antidetonante, além de elevar sua octanagem e eficiência [14].

\section{Metodologia}

\subsection{Amostras de gasolina comercial}

Foram coletadas, ao todo, seis amostras de gasolina comercial provenientes de três diferentes postos de combustíveis localizados em Santa Cruz do Sul, RS (Tabela 1).

Tabela 1 - Identificação das amostras de gasolina utilizadas no estudo.

\begin{tabular}{cc}
\hline Código da amostra & Tipo de gasolina \\
\hline GA_P1 & Aditivada (posto 1) \\
GA_P2 & Aditivada (posto 2) \\
GC_P1 & Comum (posto 1) \\
GC_P2 & Comum (posto 2) \\
GC_P3 & Comum (posto 3) \\
GPr_P2 & Premium (posto 2) \\
\hline
\end{tabular}

\subsection{Amostras de calibração}

As amostras utilizadas na calibração multivariada foram produzidas a partir da amostra de gasolina comum (GC_P3), da qual o etanol anidro foi extraído por uma solução de $\mathrm{NaCl}(10 \%$, $\mathrm{m} / \mathrm{v}$ ), conforme descrito na NBR 13992:2015 [14]. Posteriormente, foram produzidas diferentes misturas de etanol anidro e gasolina, em duplicata, resultando em uma curva analítica com concentrações de etanol entre 15 e $35 \%$, v/v (Tabela 2).

Tabela 2 - Identificação das amostras de gasolina produzidas para a construção do modelo de calibração.

\begin{tabular}{cccc}
$\begin{array}{c}\text { Código da } \\
\text { amostra }\end{array}$ & $\begin{array}{c}\text { Conc. de etanol } \\
(\boldsymbol{\%}, \mathbf{v} / \mathbf{v})\end{array}$ & $\begin{array}{c}\text { Código da } \\
\text { amostra }\end{array}$ & $\begin{array}{c}\text { Conc. de etanol } \\
(\boldsymbol{\%}, \mathbf{v} / \mathbf{v})\end{array}$ \\
\hline GP1a & 15 & GP4b & 25 \\
GP1b & 15 & GP5a & 27 \\
GP2a & 20 & GP5b & 27 \\
GP2b & 20 & GP6a & 30 \\
GP3a & 23 & GP6b & 30 \\
GP3b & 23 & GP7a & 35 \\
GP4a & 25 & GP7b & 35 \\
\hline
\end{tabular}

\subsection{Determinação do teor de álcool}

Para determinação da concentração de etanol nas amostras de gasolina foi utilizada a metodologia descrita na NBR 13992:2015 (NBR:13992). Assim, 50 mL de solução aquosa de
$\mathrm{NaCl} 10 \%$ foram adicionados em $50 \mathrm{~mL}$ de cada amostra comercial de gasolina, em duplicata, em uma proveta de $100 \mathrm{~mL}$. A proveta foi, então, invertida 10 vezes e deixada em repouso por 15 minutos, a fim de separar completamente as duas fases formadas. O volume final de fase aquosa foi observado e calculou-se o teor de etanol através da Equação 1.

$$
\mathrm{AEAC}=[(\mathrm{A}-50) \times 2]+1
$$

Equação 1 - Equação para determinação do teor de álcool etílico anidro combustível $($ AEAC) em gasolina, \% (v/v). A= volume final da fase aquosa.

\subsection{Aquisição dos espectros no infravermelho}

Os espectros, em duplicata, foram obtidos em um espectrômetro Perkin Elmer, Spectrum 400, com acessório de Refletância Total Atenuada (ATR), resolução de $4 \mathrm{~cm}^{-1}$ e 16 varreduras, na região do infravermelho médio (FTIR), na faixa de 4000 a $650 \mathrm{~cm}^{-1}$.

\subsection{Construção do modelo de PLS}

O modelo de PLS (full spectrum) foi construído através do software Chemoface versão 1.5 (http://ufla.br/chemoface/) empregando a faixa espectral analisada $\left(4000-650 \mathrm{~cm}^{-1}\right)$. A planilha, contendo dados espectrométricos, foi utilizada como matriz $\mathrm{X}_{(14 \times 3351)}$, e outra planilha, contendo os teores de etanol $(\%, \mathrm{v} / \mathrm{v})$, como vetor $\mathrm{Y}_{(14 \times 1)}$. Ambas as planilhas, construídas no Microsoft Excel ${ }^{\circledR}$, foram convertidas para o formato CSV antes de serem importadas para o software.

Os dados espectrais de dez amostras deste conjunto foram utilizados na calibração, validação cruzada e cálculo de RMSECV (root mean square error of cross-validation) do modelo, enquanto quatro amostras foram escolhidas como conjunto de validação, através do algoritmo de Kennard-Stone $[15,16]$, para a realização do teste de previsão e cálculo do RMSEP (root mean square error of prediction).

$$
\text { RMSEP ou RMSECV }=\sqrt{\frac{\sum_{\mathrm{i}=1}^{\mathrm{n}}\left(\mathrm{y}_{\mathrm{i}}-\hat{\mathrm{y}}_{\mathrm{i}}\right)^{2}}{\mathrm{n}}}
$$

Em que: $\mathrm{n}=$ número de amostras, $y_{i}=\%(\mathrm{v} / \mathrm{v})$ previsto pelo modelo; $\hat{y}_{i}=\%(\mathrm{v} / \mathrm{v})$ medido pelo método de referência.

\subsection{Análise comparativa dos métodos}

Foi realizado um teste $t$-Students com nível de significância de 0,05 nos resultados dos métodos de referência e por PLS, a fim de verificar a qualidade do modelo desenvolvido. Esta análise foi realizada no programa Microsoft Excel ${ }^{\circledR}$. 


\section{Resultados e discussões}

\subsection{Espectros de infravermelho}

Na Figura 1 estão apresentados os espectros da gasolina purificada pela extração do etanol e do etanol anidro. Como pode ser observado, as maiores diferenças entre as duas substâncias encontram-se nas regiões onde existem os picos referentes ao estiramento do $\mathrm{OH}$ (3586 a $3000 \mathrm{~cm}^{-1}$ ) e estiramentos assimétrico e simétrico de C-O (duplete em 1085 e $1048 \mathrm{~cm}^{-1}$, respectivamente), característicos da molécula de etanol e ausentes na gasolina. Nas regiões de estiramento (2968 a 2862 $\left.\mathrm{cm}^{-1}\right)$ e deformação angular (1425 a $\left.1364 \mathrm{~cm}^{-1}\right)$ do $\mathrm{CH}$, são encontrados os picos referentes às bandas vibracionais dos hidrocarbonetos presentes na molécula de gasolina, as quais encontram-se sobrepostas com os picos de etanol [3]. Essas características também foram observadas no estudo de Silva, et al. [17], ao compararem gasolinas nacionais e estrangeiras.

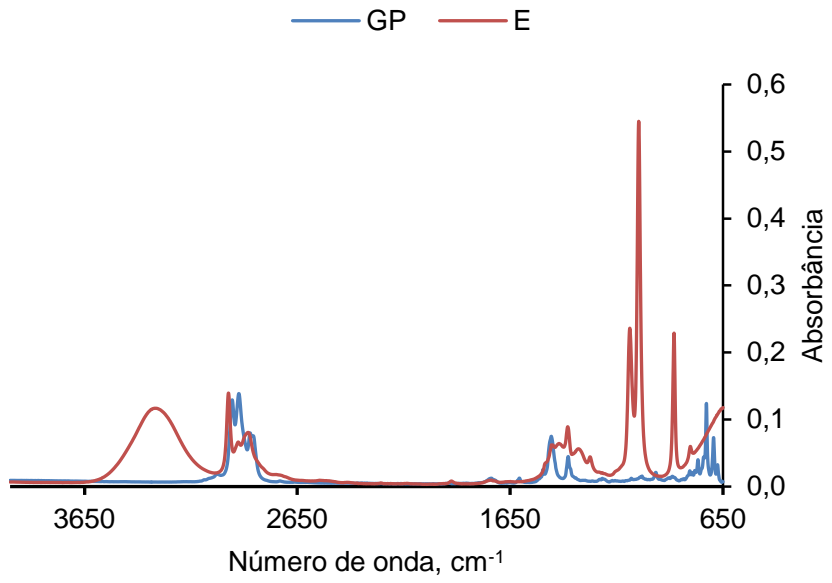

Figura 1 - Espectros de infravermelho da gasolina purificada (GP) e etanol anidro (E).

Os espectros médios das amostras de calibração estão apresentados na Figura 2. Observa-se um aumento progressivo dos valores de absorbância nas regiões características do etanol conforme aumenta a concentração do mesmo.

Os espectros de infravermelho para as amostras de gasolina comercial estão apresentados na Figura 3, e possuem características semelhantes aos espectros obtidos no estudo de [18].
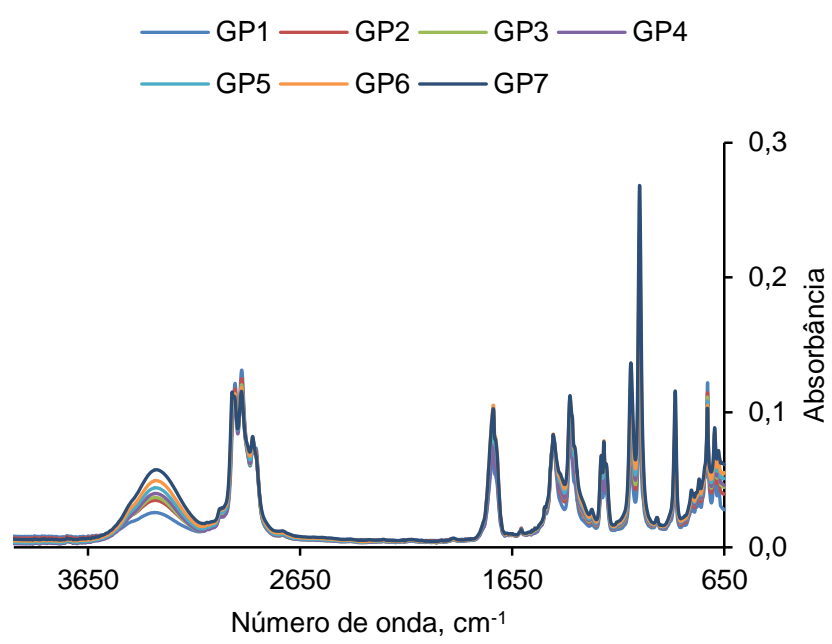

Figura 2 - Espectros médios de infravermelho das amostras de calibração.

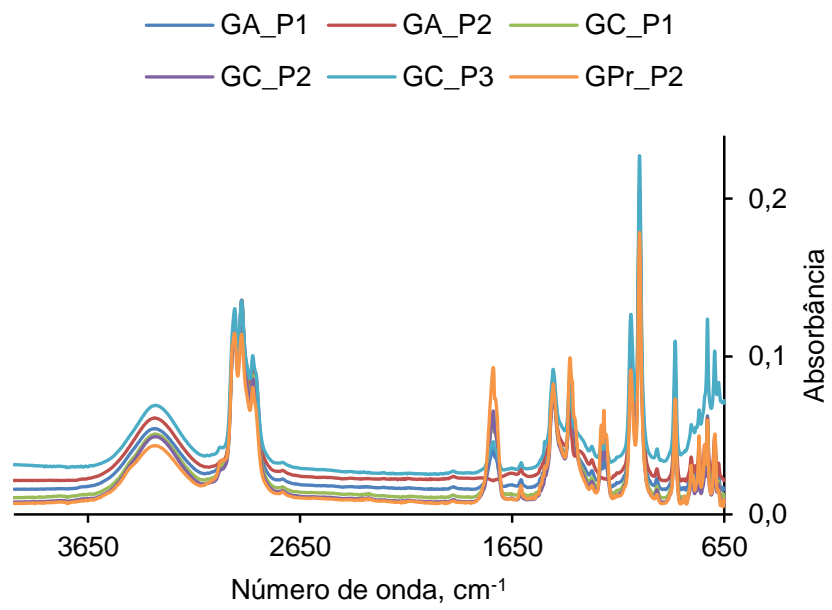

Figura 3 - Espectros de infravermelho das amostras de gasolina comercial.

\subsection{Modelo de calibração por PLS}

Para o desenvolvimento do modelo de calibração, os dados espectrais foram tratados previamente por derivada de primeira ordem e centrado na média. Com isso, foi possível a obtenção de um RMSECV mínimo de 0,66\% com 6 variáveis latentes, o que pode ser visualizado na Figura 4. 


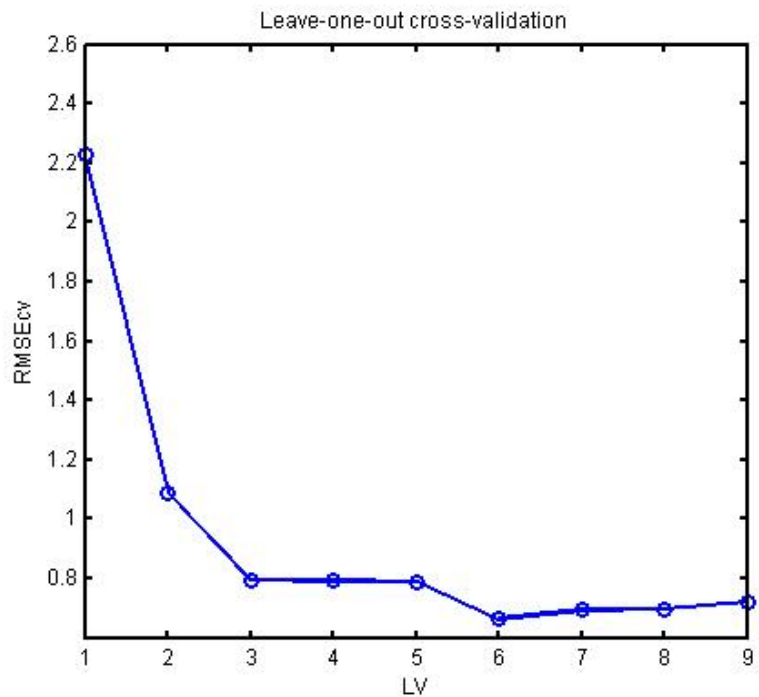

Figura 4 - Resultados de RMSECV para as possíveis combinações de variáveis latentes (LV).

Após a seleção do número de variáveis latentes a serem utilizadas na calibração, plotou-se o gráfico de correlação entre os teores de etanol adicionados às amostras e os teores preditos pelo modelo (Figura 5). O modelo obtido apresentou, além do RMSECV de $0,66 \%$ (v/v), um RMSEC de 0,17\% (v/v) e valores de 0,999 e 0,988 para os coeficientes de determinação para calibração $\left(\mathrm{R}^{2} \mathrm{c}\right)$ e validação cruzada $\left(\mathrm{R}^{2} \mathrm{cv}\right)$, respectivamente.

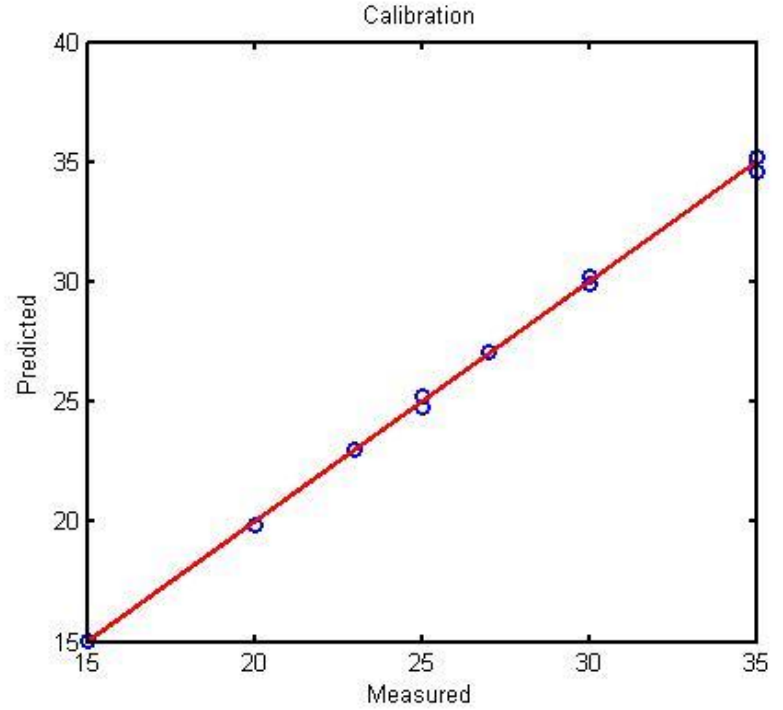

Figura 5 - Correlação entre os teores de etanol preditos (Predicted) pelo modelo e adicionados às amostras (Measured).

Na Figura 6 é possível observar a relação entre os resíduos (Studentized residuals) e a influência (Sample leverages) das amostras no modelo de calibração, podendo-se constatar a ausência de amostras anômalas no mesmo (Studentizer residuals $<2,5$, e h crítico (leverage) $<1,3$ ).

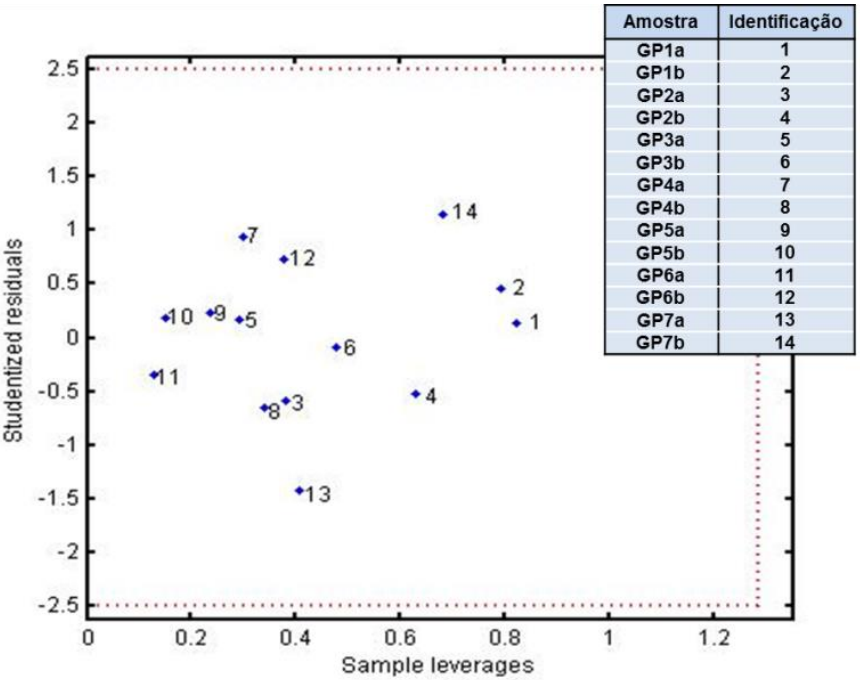

Figura 6 - Gráfico correlacionando os resíduos (Studentized residuals) e a influência das amostras no modelo (Sample leverage).

\subsection{Validação do modelo de PLS}

A Figura 8 apresenta o gráfico da correlação entre os valores preditos pelo modelo e medidos experimentalmente.

Para o conjunto de validação obteve-se um RMSEP de 0,54\% (v/v), valor próximo ao obtido por Oliveira, et al. [19], satisfazendo o resultado obtido neste estudo, e um coeficiente de determinação para predição $\left(\mathrm{R}^{2}\right.$ pred) de 0,985 . Outros estudos como os de Oliveira, et al. [19] Fernandes, et al. [20], utilizando uma região menor do infravermelho $\left(9050-6000 \mathrm{~cm}^{-1}\right)$ na detecção de adulterações na gasolina, obtiveram valores de RMSEP da ordem de $0,83 \%$ (v/v).

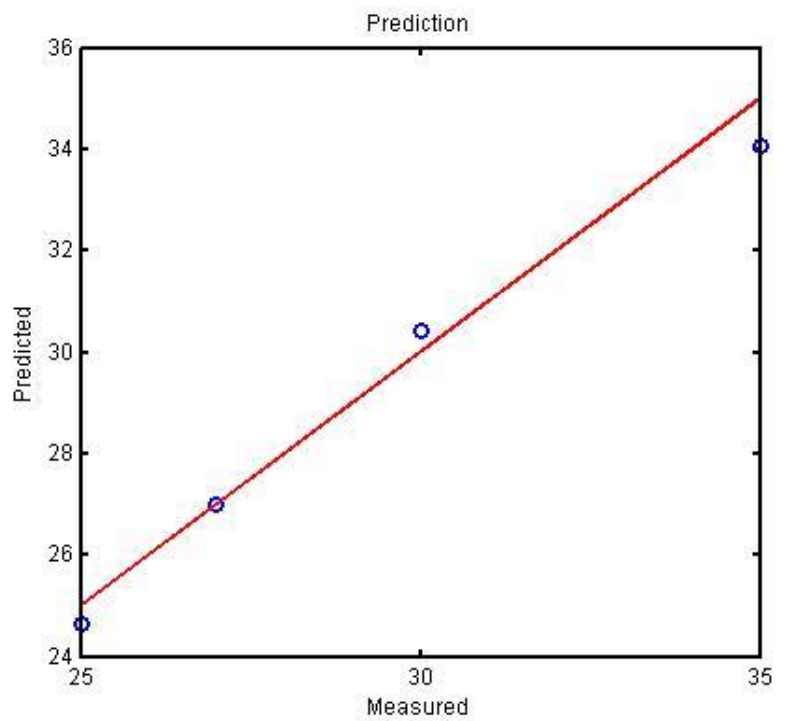

Figura 7 - Correlação entre os teores de etanol preditos (Predicted) pelo modelo e medidos pelo método de referência (Measured). 


\subsection{Teste com amostras de gasolina comercial}

Os resultados para a determinação do teor de etanol anidro combustível, nas amostras de gasolina comercial, pelo método de referência e pelo método multivariado, utilizado neste trabalho, estão sumarizados na Tabela 3.

Tabela 3 - Resultados de concentração (\%,v/v) de etanol na gasolina pelos métodos utilizados.

\begin{tabular}{cccc}
\hline Amostra & $\begin{array}{c}\text { Método de } \\
\text { referência }\end{array}$ & $\begin{array}{c}\text { Método } \\
\text { multivariado }\end{array}$ & Exatidão \\
\hline GA_P1 & $26,8 \pm 0,3, \mathrm{n}=2$ & 26,2 & 97,8 \\
GA_P2 & $25,9 \pm 2,0, \mathrm{n}=2$ & 26,1 & 100,8 \\
GC_P1 & $25,4 \pm 2,0, \mathrm{n}=2$ & 25,0 & 98,4 \\
GC_P2 & $26,8 \pm 0,3, \mathrm{n}=2$ & 29,0 & 108,2 \\
GC_P3 & $26,8 \pm 0,3, \mathrm{n}=2$ & 27,0 & 100,7 \\
GPr_P2 & $23,7 \pm 0,2, \mathrm{n}=2$ & 24,2 & 102,1 \\
\hline
\end{tabular}

Utilizando o teste $t$ Students nos dados obtidos foi possível verificar que não existe diferença significativa entre o método estabelecido pela NBR 13993:1997 e o modelo de PLS desenvolvido $(\mathrm{p}>0,05)$.

Estes resultados comprovaram que, apesar de simples e de ter sido executada com um software gratuito para análise multivariada de dados, a metodologia adotada permitiu a quantificação de etanol, em amostras de gasolina comercial, com bons resultados de exatidão $(97,8-108,2 \%)$. Além disso, a metodologia desenvolvida caracteriza-se pela alta frequência analítica (>30 amostras por hora), baixo consumo amostra (2 $\mathrm{mL})$, e de energia $\left(340 \mathrm{~W} \mathrm{~h}^{-1}\right)$ e por dispensar o uso de reagentes.

\section{Considerações Finais}

Os resultados apresentados neste trabalho demonstram que a utilização do método de regressão por mínimos quadrados parciais (PLS), associados à espectroscopia no infravermelho médio, permite a determinação de etanol anidro em gasolina, apresentando resultados equivalentes aos obtidos pelo método de referência.

Apesar de exigir maiores conhecimentos específicos e a disponibilidade de instrumentação analítica mais sofisticada que metodologia de referência, o método desenvolvido neste estudo se destaca pela elevada frequência analítica, por consumir uma quantidade até 25 vezes menor de amostra, que, por consequência, resulta numa menor geração de resíduos e numa maior frequência de análise.

\section{Agradecimentos}

Os autores agradecem ao Conselho Nacional de Desenvolvimento Científico e Tecnológico (CNPq), a Coordenação de Aperfeiçoamento de Pessoal de Nível Superior (CAPES), a Fundação de Amparo à Pesquisa do Estado do Rio Grande do Sul (FAPERGS) e ao Fundo de Apoio a Pesquisa da
Universidade de Santa Cruz do Sul (FAP), pelo apoio no desenvolvimento desta pesquisa.

\section{DETERMINATION OF ETHANOL CONTENT IN GASOLINE: DIDACTIC EXPERIMENT OF CHEMOMETRY FOR MULTIVARIATE CALIBRATION USING MID-INFRARED SPECTROSCOPY}

\section{ABSTRACT:}

The main objective of this study was to develop a multivariate partial squares (PLS) calibration model to quantify anhydrous ethanol content in commercial gasoline samples using free Chemometrics software. For this, were acquired samples' mid infrared (MIR) spectra in an experimental activity of undergraduate chemistry students, to improve the understanding of multivariate calibration methods. The PLS calibration model and the reference method $(\mathrm{ABNT})$ showed similar results $(\mathrm{p}>$ 0.05 ) in the determination of the ethanol content between 24.2 and $29.0 \%$. In addition, the method developed in this study stands out for consuming up to 25 times less sample $(2 \mathrm{ml})$, which results in less waste generation and higher frequency of analysis (> 30 samples per hour), low energy consumption (340 $\mathrm{Wh}^{-1}$ ) and without use of reagents.

Keywords: Chemometrics. PLS. Ethanol. Gasoline.

\section{Referências}

[1] DE BARROS NETO, B. et al.; 25 anos de quimiometria no Brasil, Química Nova, Vol. 29, n. 6, p. 1401, 2006.

[2] FERREIRA, M. M. C.; Quimiometria-Conceitos, Métodos e Aplicações, Campinas, SP: Editora Unicamp, Vol., n., p., 2015.

[3] FERREIRA, M. M. C. et al:; Quimiometria I: calibração multivariada, um tutorial, Química Nova, Vol. 22, n. 5, p. 724$731,1999$.

[4] RINNAN, A. et al.; Review of the most common preprocessing techniques for near-infrared spectra, TrAC Trends in Analytical Chemistry, Vol. 28, n. 10, p. 1201-1222, 2009.

[5] SOUZA, A. M. D. et al.; Teaching experiment of chemometrics for multivariate calibration in determination of paracetamol in commercial tablets using near-infrared spectroscopy: a tutorial, part II, Química Nova, Vol. 36, n. 7, p. 1057-1065, 2013.

[6] MORGANO, M. A. et al.; Determinação de açúcar total em café cru por espectroscopia no infravermelho próximo e regressão por mínimos quadrados parciais, Química Nova, Vol. 30, n. 2, p. 346-350, 2007.

[7] BRAGA, J. W.; POPPI, R. J.; Validação de modelos de calibração multivariada: uma aplicação na determinação de pureza polimórfica de carbamazepina por espectroscopia no 
infravermelho próximo, Quim. Nova, Vol. 27, n., p. 1004-1011, 2004.

[8] FRANCESQUETT, J. Z. et al.; Determinacao do poder calorifico de amostras de gasolina utilizando espectroscopia no infravermelho proximo e regressao multivariada, Orbital-The Electronic Journal of Chemistry, Vol. 5, n. 2, p. 88-95, 2013.

[9] MENEZES, C. M. et al.; Direct determination of tannins in Acacia mearnsii bark using near-infrared spectroscopy, Analytical Methods, Vol. 6, n. 20, p. 8299-8305, 2014.

[10] SOUZA, J. S.; FERRÃO, M. F.; Aplicações da espectroscopia no infravermelho no controle de qualidade de medicamentos contendo diclofenaco de potássio. Parte I: dosagem por regressão multivariada, Brazilian Journal of Pharmaceutical Sciences, Vol. 42, n. 3, p. 437-445, 2006.

[11] GUARIEIRO, L. L. N. et al.; Metodologia analítica para quantificar o teor de biodiesel na mistura biodiesel: diesel utilizando espectroscopia na região do infravermelho, Vol., n., p., 2008.

[12] TAVARES, K. M. et al.; Espectroscopia no infravermelho médio e análise sensorial aplicada à detecção de adulteração de café torrado por adição de cascas de café, Quim. Nova, Vol. 35, n. 6, p. 1164-1168, 2012.

[13] MAPA; Fixa, o percentual obrigatório de adição de etanol anidro combustível à gasolina., in: P.e.A. Ministério da Agricultura (Ed.) Portaria 75, 2015.

[14] DAZZANI, M. et al.; Explorando a Química na Determinação do Teor de álcool na Gasolina, Química Nova na Escola, No17, Vol., n., p. 42, 2003.

[15] KENNARD, R. W.; STONE, L. A.; Computer aided design of experiments, Technometrics, Vol. 11, n. 1, p. 137-148, 1969.

[16] COSTAGLIOLA, M. A. et al.; Combustion efficiency and engine out emissions of a SI engine fueled with alcohol/gasoline blends, Applied energy, Vol. 111, n., p. 1162-1171, 2013.

[17] SILVA, N. C. D. et al.; Classification of Brazilian and foreign gasolines adulterated with alcohol using infrared spectroscopy, Forensic Science International, Vol. 253, n., p. 3342, 2015.

[18] TEIXEIRA, L. S. G. et al.; Multivariate calibration in Fourier transform infrared spectrometry as a tool to detect adulterations in Brazilian gasoline, Fuel, Vol. 87, n. 3, p. 346352, 2008.

[19] OLIVEIRA, F. C. C. et al.; A escolha da faixa espectral no uso combinado de métodos espectroscópicos e quimiométricos, Química Nova, Vol. 27, n. 2, p. 218-225, 2004.

[20] FERNANDES, H. L. et al.; Simultaneous determination of methanol and ethanol in gasoline using NIR spectroscopy: Effect of gasoline composition, Talanta, Vol. 75, n. 3, p. 804-810, 2008. 